\title{
Fatostatin suppresses growth and enhances apoptosis by blocking SREBP-regulated metabolic pathways in endometrial carcinoma
}

\author{
SHUHONG GAO ${ }^{1,2}$, ZHENGZHENG SHI $^{3}$, XIN LI $^{4}$, WENZHI LI ${ }^{1}$, YILING WANG ${ }^{1}$, ZHIMING LIU $^{1}$ and JIE JIANG ${ }^{1}$ \\ ${ }^{1}$ Department of Obstetrics and Gynecology, Qilu Hospital of Shandong University, Jinan, Shandong 250012; \\ ${ }^{2}$ Department of Obstetrics and Gynecology, The Affiliated Hospital of Binzhou Medical University, Binzhou, Shandong 256603; \\ ${ }^{3}$ Department of Gynecology, The First Affiliated Hospital of Wenzhou Medical University, Wenzhou, Zhejiang 325000; \\ ${ }^{4}$ Department of Gynecology, Zaozhuang Municipal Hospital, Zaozhuang, Shandong 277000, P.R. China
}

Received August 6, 2017; Accepted January 31, 2018

DOI: $10.3892 /$ or.2018.6265

\begin{abstract}
Fatostatin, a chemical inhibitor of the sterol regulatory element-binding protein (SREBP) pathway, has been reported to possess high antitumor activity against prostate and pancreatic cancer. The main aim of the present study was to investigate the effects and mechanism of fatostatin in endometrial carcinoma (EC). In the present study, we determined that fatostatin inhibited EC cell viability and colony formation capacity, decreased the invasive and migratory capacities of EC cells, induced EC cell cycle arrest at the G2/M phase and stimulated caspase-mediated apoptosis of EC cells. In addition, fatostatin significantly decreased the protein expression levels of nuclear SREBPs and their downstream genes and increased the protein expression levels of cleaved caspase-9, caspase-3 and PARP in EC cells. In addition, the mRNA expression levels of SREBP-controlled downstream genes were also significantly downregulated. The quantification assays of fatty acids and total cholesterol revealed that the levels of free fatty acids and total cholesterol in EC cells were decreased. The present study indicated that fatostatin exhibited antitumor effects by blocking SREBP-regulated metabolic pathways and inducing caspase-mediated apoptosis in EC and may be a potent therapeutic strategy for the treatment of EC.
\end{abstract}

\section{Introduction}

Endometrial carcinoma (EC) is one of the most common gynecological malignant tumors in western countries. EC is the fourth most common cancer in women, accounting for $7 \%$

Correspondence to: Professor Jie Jiang, Department of Obstetrics and Gynecology, Qilu Hospital of Shandong University, 107 Wenhua Xi Road, Jinan, Shandong 250012, P.R. China

E-mail: qljiangjie@sdu.edu.cn

Key words: fatostatin, SREBP, endometrial carcinoma, lipid metabolism, caspase of all cancers in women in the US (1). The American Cancer Society estimated that 61,380 new cases of EC and 10,920 deaths from EC will occur in 2017 (1). Surgery and adjuvant chemo-radiotherapy are the first-line therapies used for most patients with EC (2). However, for patients with advanced or relapsed disease, and for patients who want to retain reproductive function, hormonal regimens, including progestins, luteinizing hormone releasing hormone agonists, anti-estrogens and aromatase inhibitors, are mainly applied. Additionally, progestins have been used as the first option in various protocols (2). Gunderson et al analyzed the contemporary literature on women with complex atypical hyperplasia and grade $1 \mathrm{EC}$ undergoing medical management with progestins from 2004 to 2011 (3). Forty-five studies and 391 patients were included, and $34 \%$ of the endometrial hyperplasia patients and $52 \%$ of the grade $1 \mathrm{EC}$ patients failed to respond to progestins (3). A retrospective analysis was conducted by Hahn et al to analyze the response to therapy among 35 patients with early-stage grade 1 endometrioid endometrial adenocarcinoma who were treated with progestins from January 1996 to December 2006; $34.3 \%$ of the patients exhibited no response to the progestins (4). Thus, new therapeutic targets and drugs are needed.

Sterol regulatory element-binding proteins (SREBPs) are critical regulators of lipid homeostasis that function by transcriptionally activating genes that are involved in fatty acid and cholesterol homeostasis (5). In mammalian cells, three isoforms of SREBP (SREBP-1a, SREBP-1c and SREBP-2), which are coded by two genes (SREBF1 and SREBF2) have been identified (5). Studies have suggested that SREBP-1 is the main regulator of fatty acid metabolism, while SREBP-2 predominantly regulates cholesterol metabolism (5). Additionally, several studies have reported that SREBPs function as oncogenes in various malignant tumors and that SREBPs can promote tumor progression by regulating lipogenesis (6-9). Li et al demonstrated that the expression level of SREBP-1 was significantly elevated in EC compared with that in healthy endometrium and that the expression levels were positively correlated with cancer progression (10). Eberhard et al demonstrated that the silencing of SREBP-1 or inhibition of fatty acid synthase sensitized resistant tumor 
cells to death ligands (11). Therefore, these studies revealed that blocking SREBP-regulated metabolic pathways via pharmacological intervention may be a novel therapeutic approach for treating EC.

Fatostatin is a chemical inhibitor of the SREBP pathway and inhibits the maturation and nuclear translocation of SREBPs $(12,13)$. Kamisuki et al demonstrated that fatostatin increased fatty acid mobilization and oxidation and reduced lipogenesis in obese $o b / o b$ mice while exhibiting low cytotoxicity (12). In addition, Li et al revealed that fatostatin demonstrated high antitumor activity against prostate cancer by blocking SREBP-regulated metabolic pathways and androgen receptor signaling in vitro and in vivo $(14,15)$. Furthermore, Siqingaowa et al demonstrated that fatostatin decreased pancreatic cancer cell viability and proliferation (16). In our present study, we demonstrated that fatostatin suppressed EC growth and tumorigenesis by blocking SREBP-regulated metabolic pathways in EC. Our findings revealed that inhibition of SREBPs may be a potential therapeutic strategy for EC treatment.

\section{Materials and methods}

Cell lines and culture conditions. The human EC cell lines Ishikawa and HEC-1A were kindly provided by Professor Beihua Kong (Qilu Hospital, Shandong University, Jinan, China). Ishikawa cells were cultured in RPMI-1640 medium (with 10\% FBS). HEC-1A cells were cultured in Dulbecco's modified Eagle's medium (DMEM) (with 10\% FBS). The cells were cultured in a $37^{\circ} \mathrm{C}$ incubator with $5 \% \mathrm{CO}_{2}$.

Compounds. Fatostatin A (chemical name: 4-[4-(4-meth ylphenyl)-2-thiazolyl]-2-propyl-pyridine, hydrobromide; synonym: 125B11; formula: C18H18N2S) was purchased from MedChem Express (Monmouth Junction, NJ, USA). The stock solution $(10,000 \mu \mathrm{M})$ was prepared with dimethyl sulfoxide (DMSO) and stored at $-20^{\circ} \mathrm{C}$. The working concentration of fatostatin was diluted in the respective medium, and the final concentration of DMSO was $<0.1 \%(\mathrm{v} / \mathrm{v})$. The control groups were treated with an equal volume of DMSO.

Cell viability assays. We analyzed cell viability with MTT assays and determined the 50\% inhibitory concentration $\left(\mathrm{IC}_{50}\right)$. EC cells were seeded in 96-well plates at a density of $8 \times 10^{3}$ (Ishikawa) or $6 \times 10^{3}$ (HEC-1A) cells/well. Following overnight incubation, the cells were treated with either the vehicle control or different concentrations of fatostatin $(5,10,15,20$, 30,40 and $50 \mu \mathrm{M}$ for Ishikawa cells and 2.5, 5.0, 7.5, 10.0, 15.0 and $20.0 \mu \mathrm{M}$ for HEC-1A cells) for 24,48 and $72 \mathrm{~h}$. Finally, cell viability was assessed with the infinite M200 PRO (Bio-Rad Laboratories, Hercules, CA, USA) after adding $20 \mu \mathrm{l}$ of MTT into the culture medium, incubating the cells for $4 \mathrm{~h}$ at $37^{\circ} \mathrm{C}$, and dissolving the formazan product in $100 \mu \mathrm{l}$ of DMSO. Data were collected from at least three independent experiments with triplicate wells. Based on the readings, we calculated the $\mathrm{IC}_{50}$ values using GraphPad Prism 5 for further study. For the growth curve assays, cells were seeded in 96-well plates at a density of $4 \times 10^{3}$ (Ishikawa) or $3 \times 10^{3}$ (HEC-1A) cells/well and treated with either the vehicle control or different concentrations of fatostatin $(5,10$ and $20 \mu \mathrm{M}$ for Ishikawa cells and
$1.25,2.50$ and $5.00 \mu \mathrm{M}$ for HEC-1A cells) for 5 days. The OD value of each well was read daily with the infinite M200 PRO. Data were collected from at least three independent experiments with triplicate wells.

Colony formation assays. For the clonogenic assays, cells in the logarithmic growth phase (200) were seeded in 6-well plates in culture medium containing either the vehicle control or different concentrations of fatostatin $(2.5,5.0$ and $10.0 \mu \mathrm{M}$ for Ishikawa cells and $0.625,1.250$ and $2.500 \mu \mathrm{M}$ for HEC-1A cells) for two weeks. Each group was allotted three plates. The colonies that formed on each plate were stained with crystal violet, and the number of colonies was determined quantitatively with Gel-Pro Analyzer (Media Cybernetics, Inc., Rockville, MD, USA).

Invasion and migration assays. The in vitro $\mathrm{EC}$ cell invasion and migration assays were performed with 24-well Boyden chambers $(8-\mu \mathrm{m}$ pore size; Corning Costar, Cambridge, MA, USA). The undersides of the upper chambers were precoated with Matrigel (BD Biosciences, San Jose, CA, USA) for the invasion assays. Following treatment with either the vehicle control or different concentrations of fatostatin (10, 20 and $40 \mu \mathrm{M}$ for Ishikawa cells and 2.5, 5.0 and $10.0 \mu \mathrm{M}$ for HEC-1A cells) for $48 \mathrm{~h}$, Ishikawa cells (1.5x105/chamber) and HEC-1A cells $\left(1.5 \times 10^{5} /\right.$ chamber $)$ were seeded into the upper chambers. After a period of incubation ( $48 \mathrm{~h}$ for the invasion assay and $24 \mathrm{~h}$ for the migration assay), the invading or migrated cells were stained with crystal violet, images were captured with an Olympus IX51 inverted microscope and quantified using ImageJ software.

Cell cycle and apoptosis analysis. Following treatment with either the vehicle control or different concentrations of fatostatin (10, 20 and $40 \mu \mathrm{M}$ for Ishikawa cells and 2.5, 5.0 and $10.0 \mu \mathrm{M}$ for HEC-1A cells) for $48 \mathrm{~h}$, Ishikawa and HEC-1A cells were fixed, stained with a PI/RNase staining buffer (Pharmingen; BD Biosciences, San Diego, CA, USA) and analyzed with a FACS flow cytometer (BD Biosciences, Franklin Lakes, NJ, USA) on the basis of $2 \mathrm{~N}$ and $4 \mathrm{~N}$ DNA content. The data were analyzed by ModFit LT 3.2 software (Verity Software House, Topsham, ME, USA). Following treatment with either the vehicle control or fatostatin for $48 \mathrm{~h}$, cell apoptosis analysis was performed with a FACS flow cytometer using the FITC Annexin V Apoptosis Detection Kit I (Pharmingen; BD Biosciences) according to the manufacturer's instructions. The data were analyzed by CellQuest software (BD Biosciences, Franklin Lakes, NJ, USA).

Protein extraction and western blot analysis. After treatment with either the vehicle control or different concentrations of fatostatin (10,20 and $40 \mu \mathrm{M}$ for Ishikawa cells and 2.5, 5.0 and $10.0 \mu \mathrm{M}$ for HEC-1A cells) for $24 \mathrm{~h}$, the cells were collected and lysed in a mixed RIPA buffer, containing PMSF and NaF (100:1:1; Beyotime Institute of Biotechnology, Haimen, China). Protein concentrations were determined with the BCA protein assay kit (Tiangen Biotech Co., Ltd., Beijing, China). The proteins were then separated on a $10 \%$ or $12 \%$ polyacrylamide gel and transferred to a polyvinylidene difluoride (PVDF) membrane (Immobilon-P; Millipore, Bedford, MA, 
USA). Following incubation with the appropriate primary antibodies for $10-16 \mathrm{~h}$ at $4^{\circ} \mathrm{C}$ and the secondary antibodies for $2 \mathrm{~h}$ at room temperature, the protein bands were detected using horseradish peroxidase luminescence solution (Millipore, Bedford, MA, USA) and ImageQuant LAS4000 (General Electric Company, Boston, MA, USA) and quantified with ImageJ software. The GAPDH band served as the loading control. The primary antibodies used in the experiments were as follows: SREBP-1 (dilution 1:200; rabbit polyclonal; cat. no. sc-8984; Santa Cruz Biotechnology, Inc., Dallas, TX, USA); FASN (dilution 1:1,000; rabbit polyclonal; cat. no. ab96866; Abcam, Cambridge, UK); SREBP-2 (dilution 1:200; mouse monoclonal; cat. no. sc-13552); HMGCR (dilution 1:500; mouse monoclonal; cat. no. sc-271595); caspase-9 (dilution 1:500; mouse monoclonal; cat. no. sc-56076; all from Santa Cruz Biotechnology, Inc.), caspase-3 (dilution 1:500; rabbit monoclonal; cat. no. ab32042; Abcam), PARP-1 (dilution 1:500; mouse monoclonal; cat. no. sc-8007; Santa Cruz Biotechnology, Inc.); cleaved-PARP (dilution 1:1,000; rabbit monoclonal; cat. no. 5625S); and GAPDH (dilution 1:1,000; rabbit monoclonal; cat. no. 2118S; both from Cell Signaling Technology, Inc., Danvers, MA, USA). Horseradish peroxidase-conjugated secondary antibodies: m-IgGк BP-HRP (dilution 1:2,000; cat. no. sc-516102; Santa Cruz Biotechnology, Inc.) and goat anti-rabbit IgG H\&L (HRP) (dilution 1:2,000; cat. no. ab205718; Abcam) were used.

Quantitative real-time RT-PCR ( $q R T-P C R)$ analysis. Total RNA from the cells treated for $24 \mathrm{~h}$ with either the vehicle control or different concentrations of fatostatin (10, 20 and $40 \mu \mathrm{M}$ for Ishikawa cells and 2.5, 5.0 and 10.0 $\mu \mathrm{M}$ for HEC-1A cells) was extracted using TRIzol reagent (Invitrogen; Thermo Fisher Scientific, Carlsbad, CA, USA) following the manufacturer's instructions. After assessing the concentrations using the NanoPhotometer Pearl (Implen GmbH, Munich, Germany), $3 \mu \mathrm{g}$ of total RNA was reverse-transcribed into cDNA using the SuperScript ${ }^{\mathrm{TM}}$ II Reverse Transcriptase kit (Invitrogen; Thermo Fisher Scientific). qRT-PCR reactions were performed on an Applied Biosystems 7900HT Fast Real-Time PCR system with SYBR Premix Ex Taq (Tli RNaseH Plus) (cat. no. RR420A; Takara Bio, Inc., Otsu, Japan) in a $10-\mu 1$ reaction system, and GAPDH was used as the control. The primers used in the present study included ATP citrate lyase (ACL), stearoyl-CoA desaturase-1 (SCD-1), fatty acid synthase (FASN), 3-hydroxy-3-methyl-glutaryl-CoA synthase 1 (HMGCS1), 3-hydroxy-3-methyl-glutaryl-CoA reductase (HMGCR), mevalonate 5-pyrophosphate decarboxylase (MVD), mevalonate kinase (MVK), low-density lipoprotein receptor (LDLR), insulin-induced gene 1 (INSIG1), SREBP cleavage activating protein (SCAP) and GAPDH. The primer sequences are listed in Table I.

Quantification of fatty acids and total cholesterol. Following treatment with either the vehicle control or fatostatin $(20 \mu \mathrm{M}$ for Ishikawa cells and $5 \mu \mathrm{M}$ for HEC-1A cells) for $48 \mathrm{~h}$, the amounts of fatty acids and total cholesterol were assessed using a free fatty acid quantification detection kit and a total cholesterol quantification detection kit (Solarbio Science \& Technology Co., Ltd., Beijing, China) following the
Table I. The primer sequences used for qRT-PCR.

\begin{tabular}{|c|c|c|}
\hline \multirow{2}{*}{$\begin{array}{l}\text { Gene } \\
\mathrm{ACL}\end{array}$} & \multicolumn{2}{|c|}{ Sequence $\left(5^{\prime}-3^{\prime}\right)$} \\
\hline & $\mathrm{F}$ & TGTAACAGACCAGGAACCC \\
\hline & $\mathrm{R}$ & CTGTACCCCAGTGGCTGTTT \\
\hline \multirow[t]{2}{*}{ SCD-1 } & $\mathrm{F}$ & CACTTGGGAGCCCTGTATGG \\
\hline & $\mathrm{R}$ & AGCCGAGCTTTGTAAGAGCG \\
\hline \multirow[t]{2}{*}{ FASN } & $\mathrm{F}$ & CGGTACGCGACGGCTGCCTG \\
\hline & $\mathrm{R}$ & GCTGCTCCACGAACTCAAACACCG \\
\hline \multirow[t]{2}{*}{ HMGCS1 } & $\mathrm{F}$ & GAGGGCTTCGTGGGACACATA \\
\hline & $\mathrm{R}$ & GCCACTGGGATGGATCTTT \\
\hline \multirow[t]{2}{*}{ HMGCR } & $\mathrm{F}$ & GTCATTCCAGCCAAGGTTGT \\
\hline & $\mathrm{R}$ & GGGACCACTTGCTTCCATTA \\
\hline \multirow[t]{2}{*}{ MVD } & $\mathrm{F}$ & ACCACGGGGACACCACGGT \\
\hline & $\mathrm{R}$ & CCACACAGCAGCCACAAACTC \\
\hline \multirow[t]{2}{*}{ MVK } & $\mathrm{F}$ & CCTTGTGGCTGGCGTCAGAAA \\
\hline & $\mathrm{R}$ & CGAGGGCATTCAGATGGTGCT \\
\hline \multirow[t]{2}{*}{ LDLR } & $\mathrm{F}$ & CAACGGCTCAGACGAGCAAG \\
\hline & $\mathrm{R}$ & AGTCACAGACGAACTGCCGAGA \\
\hline \multirow[t]{2}{*}{ INSIG1 } & $\mathrm{F}$ & GGACGACAGTTAGCTATGGGTGTT \\
\hline & $\mathrm{R}$ & GAGTCATTTGTACAGTCAGCCCGA \\
\hline \multirow[t]{2}{*}{ SCAP } & $\mathrm{F}$ & TATCTCGGGCCTTCTACAACCA \\
\hline & $\mathrm{R}$ & АСАСААСТССТССААGСТССТG \\
\hline \multirow[t]{2}{*}{ GAPDH } & $\mathrm{F}$ & TGCACCACCAACTGCTTAGC \\
\hline & $\mathrm{R}$ & GGCATGGACTGTGGTCATGAG \\
\hline
\end{tabular}

F, forward; R, reverse.

manufacturer's instructions. The data were analyzed using the calculation formula provided in the instruction manual.

Statistical analysis. All experiments were repeated three times. The data were expressed as the mean \pm standard deviation (SD). Relative quantification of RNA expression was assessed using the $2^{-\Delta \Delta \mathrm{Ct}}$ method. All statistical analyses were performed using GraphPad Prism version 5.01. Statistically significant differences between cell viabilities were analyzed by two-way ANOVA, and comparisons of the other quantitative data were analyzed by Student's t-test. Statistical significance was defined as $\mathrm{P}<0.05$.

\section{Results}

Fatostatin inhibits EC cell viability and colony formation. To determine the effect of fatostatin on EC cells, we first investigated the effect of fatostatin on EC cell viability. We treated Ishikawa and HEC-1A EC cells with different concentrations of fatostatin for 24,48 and $72 \mathrm{~h}$, as described in Materials and methods. We found that fatostatin significantly inhibited the viability of both cell lines in a dose- and time-dependent manner. In addition, the $\mathrm{IC}_{50}$ values (72-h treatment) for fatostatin in the Ishikawa and HEC-1A cells were 17.96 and $4.53 \mu \mathrm{mol} / \mathrm{l}$, respectively (Fig. 1A and B). 


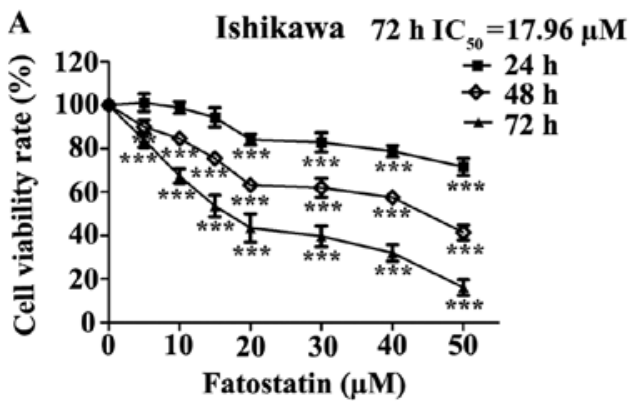

C

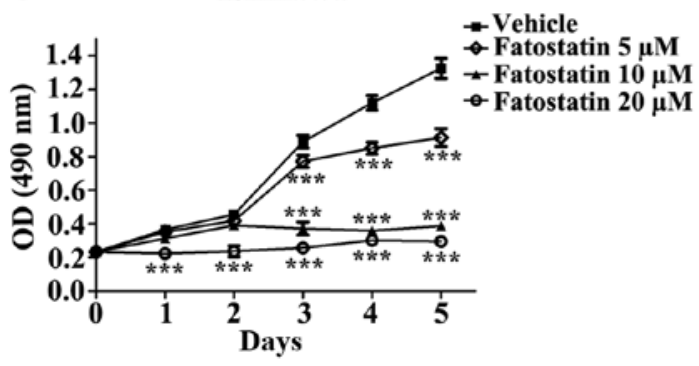

$\mathbf{E}$

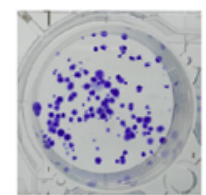

Vehicle

$\mathbf{F}$

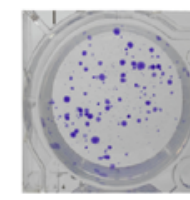

Vehicle
Ishikawa

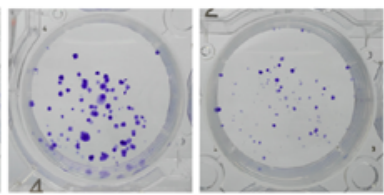

5.0

Fatostatin $(\mu \mathrm{M})$

HEC-1A

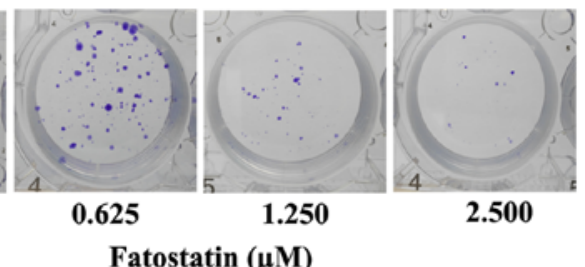

B

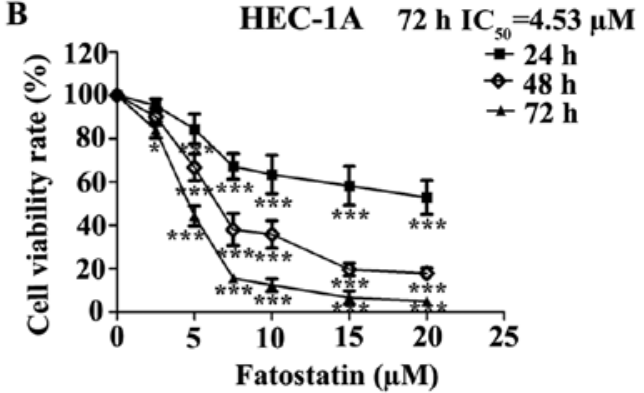

D
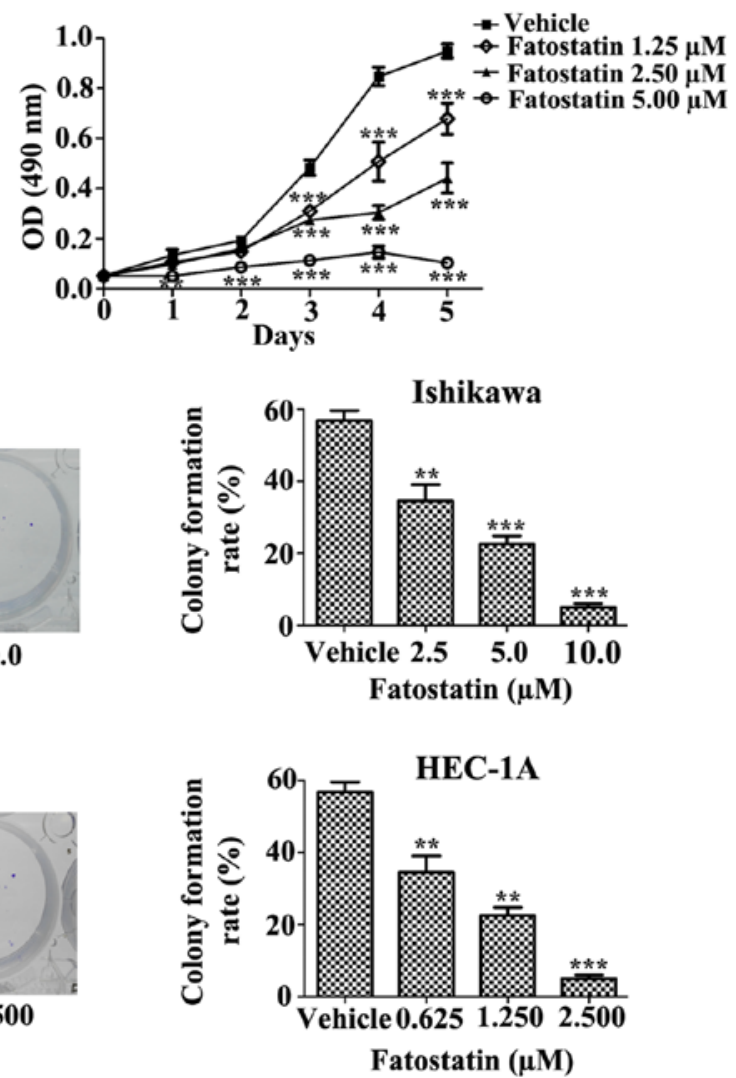

Figure 1. (A and B) Fatostatin significantly inhibited the viability of Ishikawa and HEC-1A cells. The cell viability of the vehicle-treated group was regarded as $100 \%$. The $\mathrm{IC}_{50}$ values of the 72 -h treatment are shown in the diagram. (C and D) The growth rate of each cell line was determined by MTT analysis. The OD values were positively correlated with the number of cells. Ishikawa and HEC-1A cells were notably inhibited by fatostatin in a dose- and time-dependent manner. (E and F) Fatostatin significantly inhibited the numbers and sizes of the colonies formed by Ishikawa and HEC-1A cells in a dose-dependent manner. After 2 weeks of culture, the number of colonies was determined quantitatively with Gel-Pro Analyzer. ${ }^{*} \mathrm{P}<0.05,{ }^{* *} \mathrm{P}<0.01$ and ${ }^{* * * *} \mathrm{P}<0.001$.

Then, we determined the growth rate of each cell line with or without fatostatin treatment using MTT assays. We observed that the growth rates of the Ishikawa and HEC-1A cells were notably reduced by fatostatin in a dose- and time-dependent manner (Fig. 1C and D). Furthermore, we examined the effect of fatostatin on EC cell colony formation ability. Following 2 weeks of culture, fatostatin significantly inhibited the number and size of the colonies formed in the Ishikawa and HEC-1A cells in a dose-dependent manner (Fig. 1E and F). Collectively, these results revealed that fatostatin inhibits EC cell viability and colony formation.

Fatostatin decreases the invasive and migratory capacities of EC cells. Since invasion and migration are two essential steps for malignant progression and metastasis, we examined the effects of fatostatin on the invasive and migratory capaci- ties of EC cells. We treated Ishikawa and HEC-1A cells with fatostatin at different concentrations for $48 \mathrm{~h}$ and used Boyden chambers to assess cell invasion and migration, as described in Materials and methods. We found that the number of cells invading through the membrane and the number of migrating cells were significantly decreased by fatostatin compared with those in the vehicle-treated group (Fig. 2), suggesting that fatostatin could effectively and dose-dependently inhibit the invasive and migratory capacities of both cell lines.

Fatostatin induces G2/M cell cycle arrest and caspase-dependent apoptosis in EC cells. Cell viability is tightly controlled by the cell cycle. Therefore, we examined the effects of fatostatin treatment on cell cycle distribution. Following treatment for $48 \mathrm{~h}$ with either the vehicle control or fatostatin at different concentrations, the percentage of cells in each cell cycle phase 
A
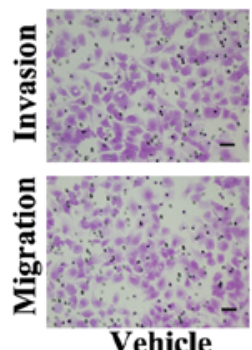

Vehicle

B

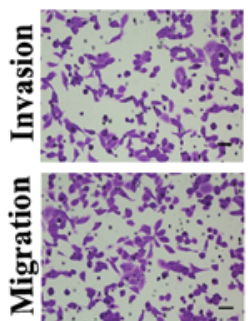

Vehicle
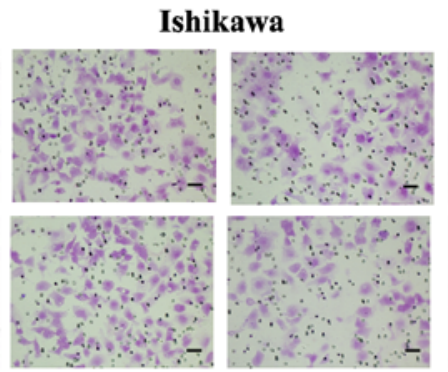

10

Fatostatin $(\mu \mathrm{M})$

HEC-1A

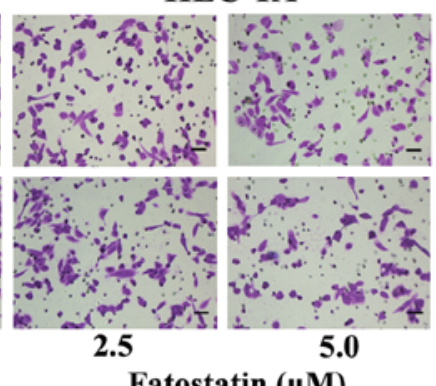

Fatostatin $(\mu \mathrm{M})$

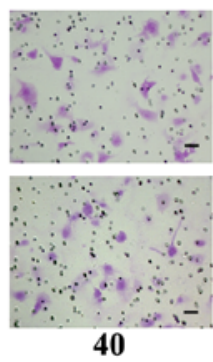

40

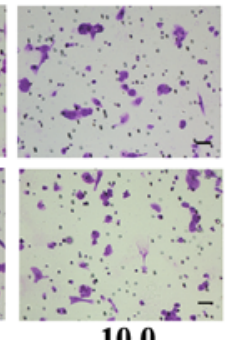

10.0

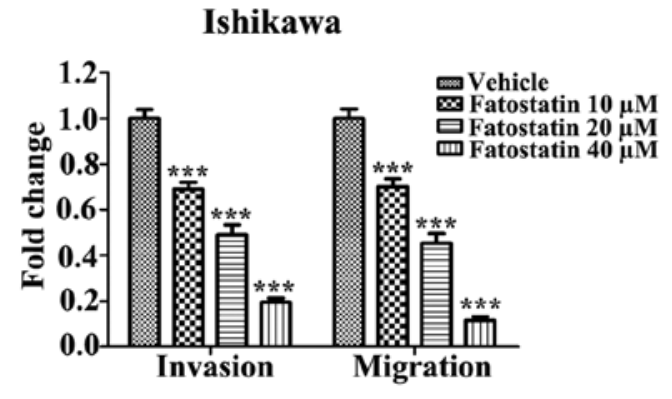

HEC-1A

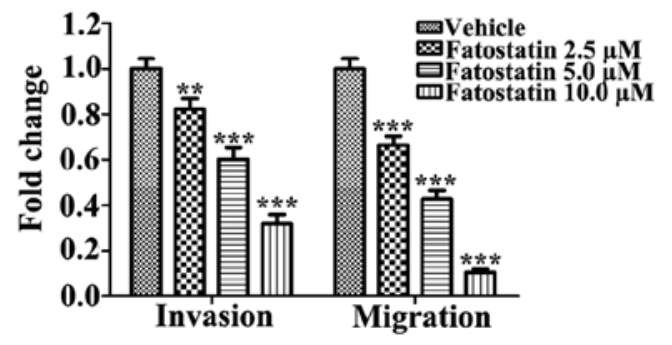

Figure 2. Fatostatin effectively inhibited the invasive and migratory capacities of Ishikawa and HEC-1A cells in a dose-dependent manner (x200 magnification). The number of cells invading through the membrane and the number of migrating cells in the vehicle-treated group were regarded as 1.0 . Bars, $30 \mu \mathrm{m}$. ${ }^{* *} \mathrm{P}<0.01$ and ${ }^{* * *} \mathrm{P}<0.001$.
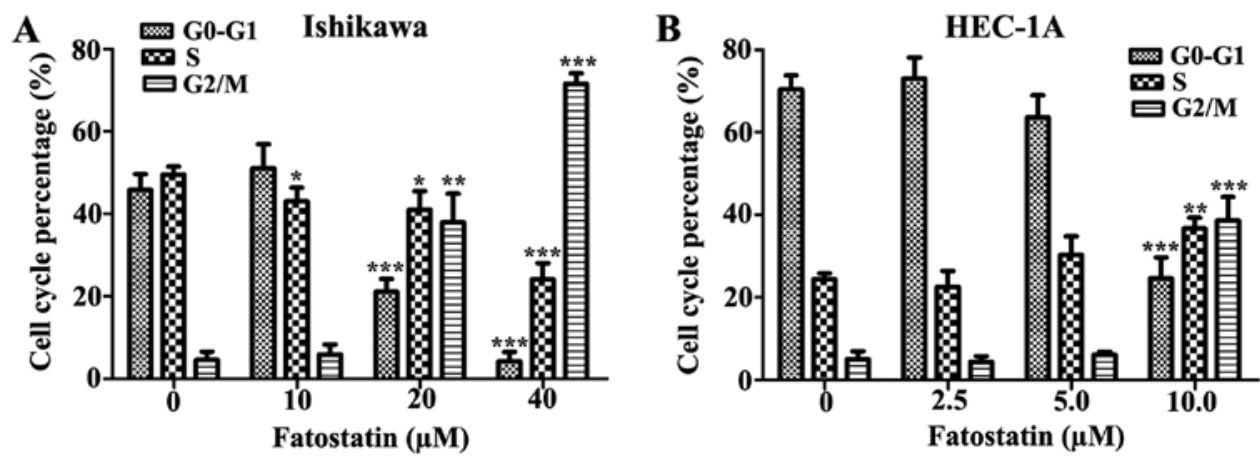

Figure 3. Fatostatin induced a significant decrease in the percentage of cells in the $\mathrm{G}_{0} / \mathrm{G}_{1}$ phase and a significant increase in the percentage of cells in the $\mathrm{G} 2 / \mathrm{M}$ phase in both Ishikawa $(20$ and $40 \mu \mathrm{M})$ and HEC-1A $(10 \mu \mathrm{M})$ cells. ${ }^{*} \mathrm{P}<0.05,{ }^{* *} \mathrm{P}<0.01$ and ${ }^{* * *} \mathrm{P}<0.001$.

was determined by PI-staining-based flow cytometry. The data revealed that fatostatin induced a significant decrease in the percentage of cells in the $\mathrm{G}_{0} / \mathrm{G}_{1}$ phase and a significant increase in the percentage of cells in the $\mathrm{G} 2 / \mathrm{M}$ phase for both Ishikawa (20 and $40 \mu \mathrm{M})$ and HEC-1A $(10 \mu \mathrm{M})$ cells. In the Ishikawa cells, the number of cells in the $\mathrm{S}$ phase was decreased by fatostatin treatment, while in the HEC-1A cells, the number of cells was increased (Fig. 3). Therefore, fatostatin promoted a significant accumulation of both Ishikawa and HEC-1A cells in the $\mathrm{G} 2 / \mathrm{M}$ phase.

To explore whether fatostatin induced apoptosis, the level of apoptosis was examined by flow cytometry using the FITC Annexin V Apoptosis Detection Kit I after treatment with either the vehicle control or fatostatin at different concentrations for $48 \mathrm{~h}$. The data revealed that the number of apoptotic cells was notably increased with fatostatin treatment at higher concentrations [Ishikawa (20 and $40 \mu \mathrm{M})$ and HEC-1A
(10 $\mu \mathrm{M})]$ (Fig. 4A-C). In the Ishikawa cells, the number of early apoptotic cells was significantly increased by fatostatin at both treatment concentrations (20 and $40 \mu \mathrm{M})$ (Fig. 4D), while the number of late apoptotic cells was increased significantly only by the higher concentration of fatostatin (40 $\mu \mathrm{M})$ (Fig. 4E). In the HEC-1A cells, the number of early apoptotic cells was significantly increased with fatostatin treatment $(10 \mu \mathrm{M})($ Fig. 4F), while the number of late apoptotic cells was only mildly increased, showing no statistical significance $(\mathrm{P}=0.27)$ (Fig. 4G). In addition, to further define the mechanism underlying fatostatin-induced apoptotic cell death, we determined the expression levels of caspases by western blot analysis. Fatostatin decreased the expression of full-length caspase-9, caspase-3 and PARP and increased the expression of cleaved caspase-9, caspase-3 and PARP in Ishikawa and HEC-1A cells (Fig. 5). These results indicated that fatostatin induced caspase-dependent apoptotic death in EC cells. 

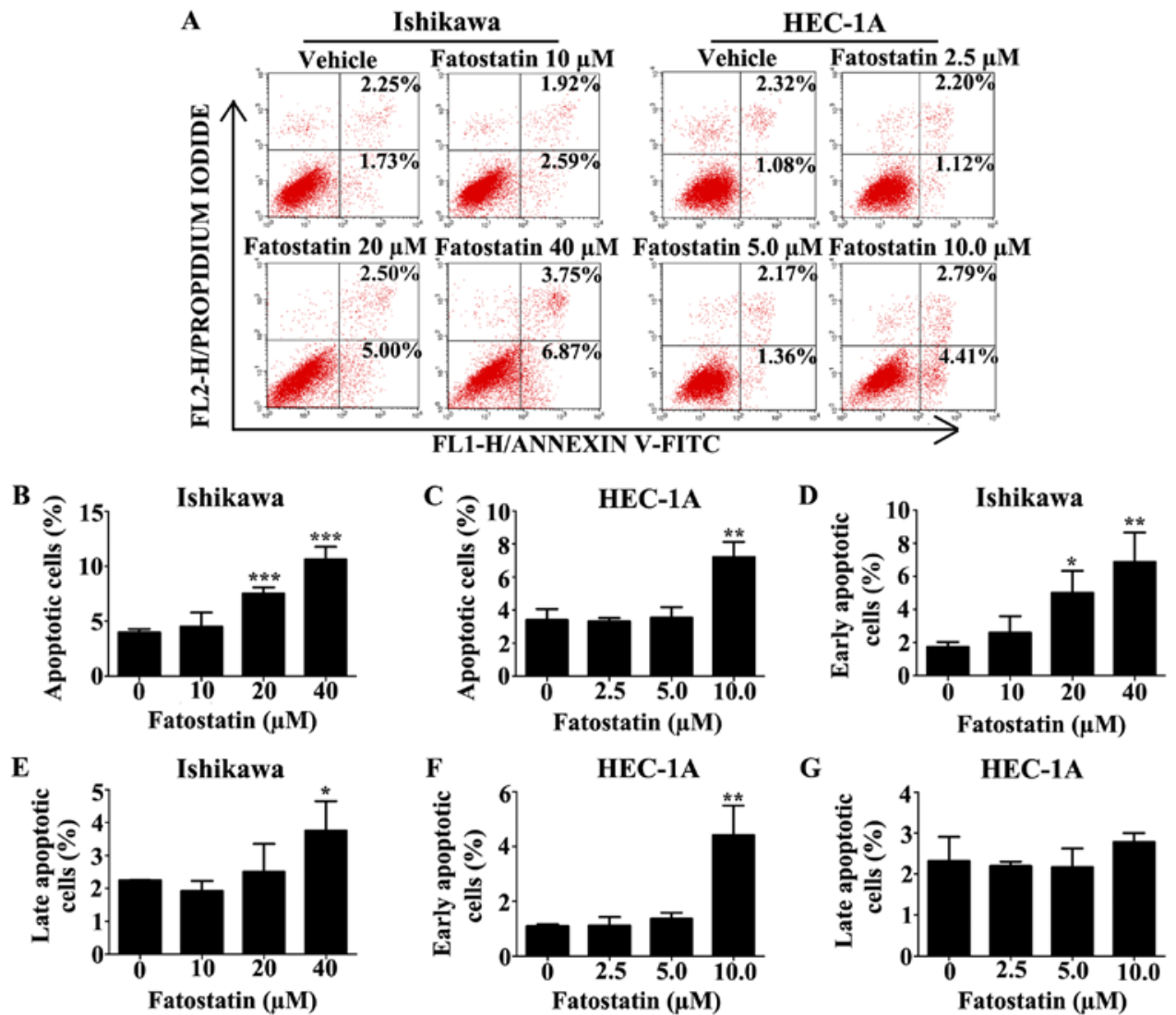

Figure 4. (A-C) The number of apoptotic cells was notably increased with the higher concentrations of fatostatin treatment [Ishikawa $(20$ and $40 \mu \mathrm{M})$ and HEC-1A $(10 \mu \mathrm{M})]$. (D) The number of early apoptotic cells was significantly increased with fatostatin treatment $(20$ and $40 \mu \mathrm{M})$ in Ishikawa cells. (E) The number of late apoptotic cells was significantly increased only by fatostatin at $40 \mu \mathrm{M}$ in Ishikawa cells. (F) The number of early apoptotic cells was significantly increased with fatostatin treatment $(10 \mu \mathrm{M})$ in HEC-1A cells. (G) The number of late apoptotic cells was only mildly increased in HEC-1A cells (P=0.27). " $\mathrm{P}<0.05$, ${ }^{* * *} \mathrm{P}<0.01$ and ${ }^{* * * *} \mathrm{P}<0.001$.

A

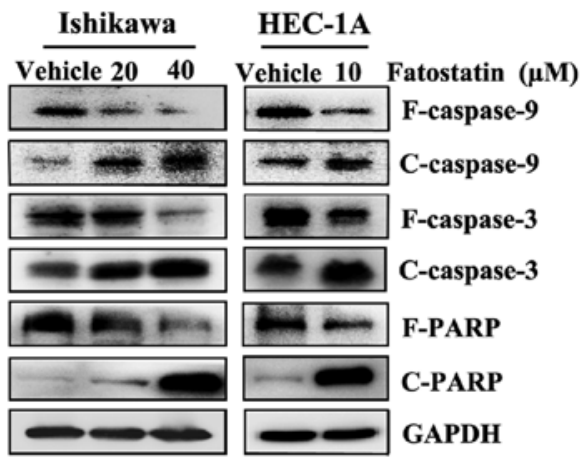

D

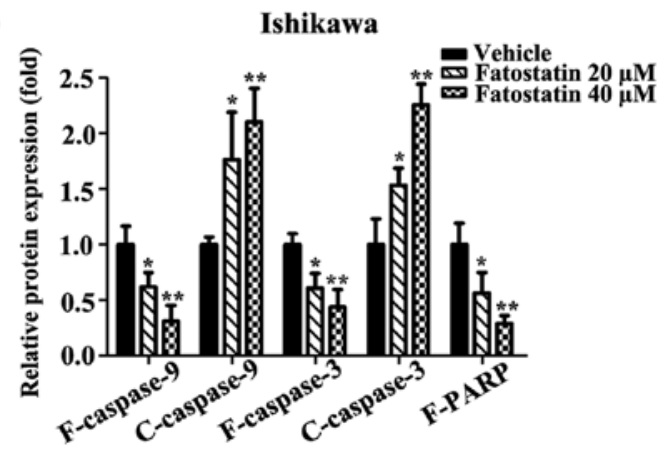

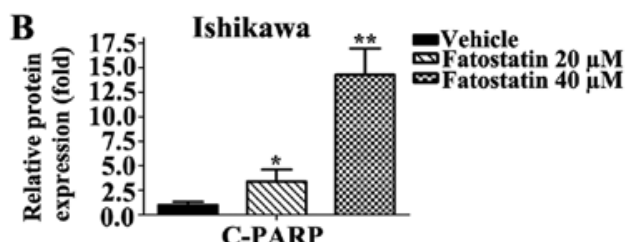

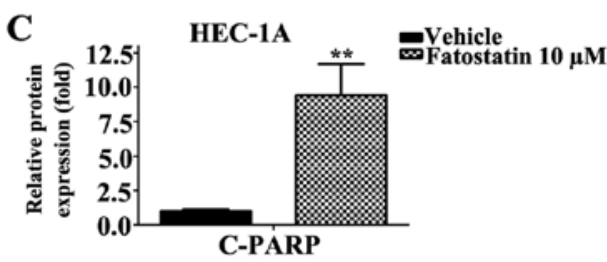

E

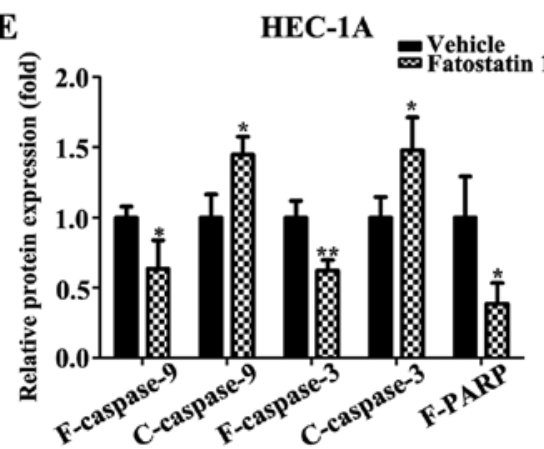

Figure 5. Fatostatin decreased the expression of the full-length caspase-9, caspase-3 and PARP and increased the expression of the cleaved caspase-9, caspase-3 and PARP in Ishikawa and HEC-1A cells. (A) The bands and (B-E) the results of the semi-quantification from the western blot analysis are shown. The GAPDH band served as the loading control. The bands of the vehicle-treated group were regarded as 1.0 . $^{*} \mathrm{P}<0.05$ and ${ }^{* * *} \mathrm{P}<0.01$. 
A
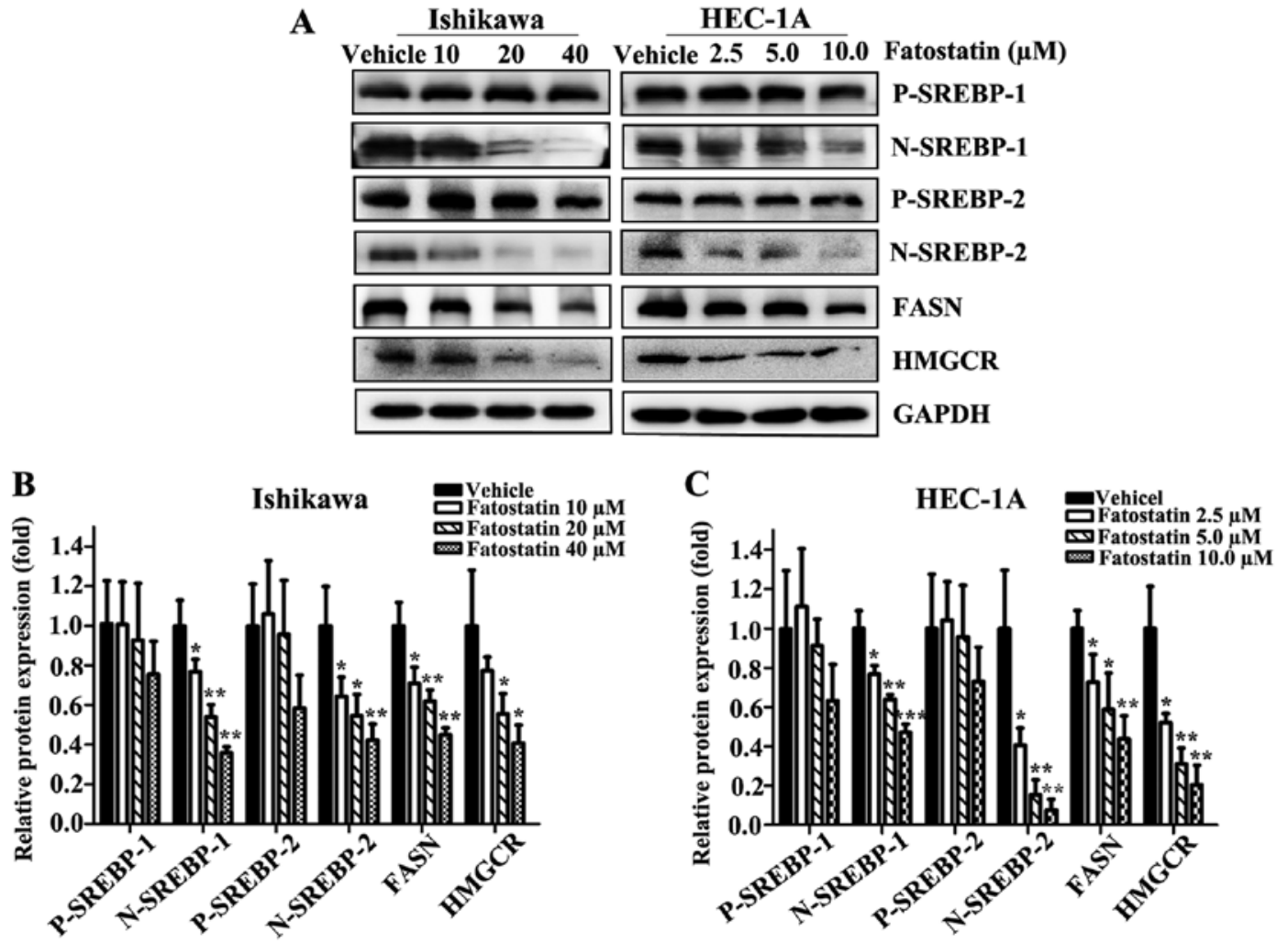

Figure 6. The precursor and nuclear SREBPs and downstream proteins FASN and HMGCR were examined by western blotting in Ishikawa and HEC-1A cells after treatment with fatostatin for $24 \mathrm{~h}$. The expression levels of the nuclear SREBPs, FASN and HMGCR were significantly decreased in a dose-dependent manner, but the precursor SREBPs were only mildly altered exhibiting no statistical significance. (A) The protein bands and (B-C) the results of the semi-quantification of the western blot analysis are shown. The GAPDH band served as the loading control. The bands of the vehicle-treated group were regarded as 1.0. ${ }^{*} \mathrm{P}<0.05,{ }^{* *} \mathrm{P}<0.01$ and ${ }^{* * *} \mathrm{P}<0.001$.
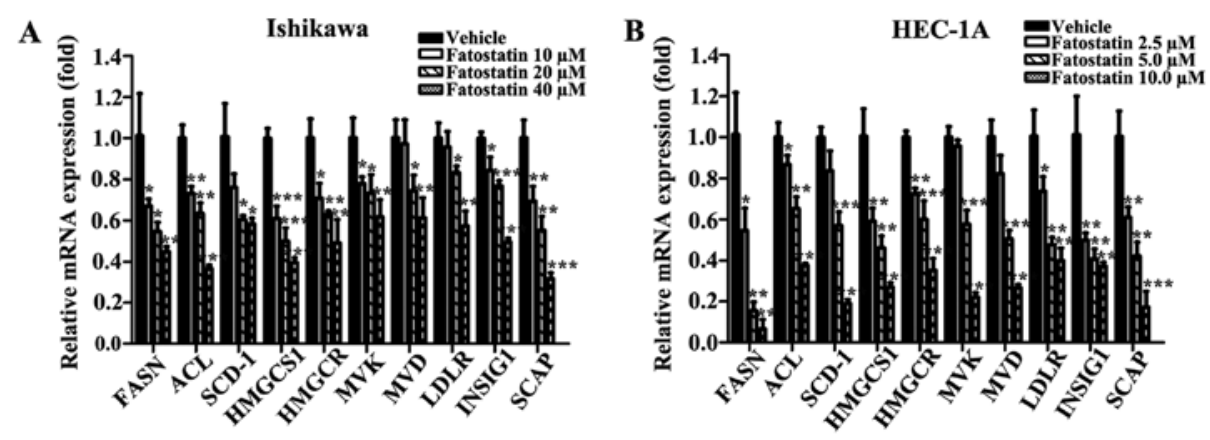

Figure 7. mRNA expression of SREBP-controlled anabolic genes was examined by qRT-PCR, and the mRNA expression levels of these genes were significantly downregulated in the fatostatin-treated cells compared with those in the vehicle-treated cells. The mRNA expression levels of the vehicle-treated group were regarded as 1.0. ${ }^{*} \mathrm{P}<0.05,{ }^{* *} \mathrm{P}<0.01$ and ${ }^{* * * *} \mathrm{P}<0.001$.

Fatostatin significantly inhibits SREBP metabolic pathways and decreases free fatty acid and total cholesterol levels in EC cells. Fatostatin is a chemical inhibitor of the SREBP pathway and directly interacts with SCAP at a distinct domain from the sterol-binding site and blocks the endoplasmic reticulum (ER) exit of SCAP and the ER-to-Golgi transport of SREBPs $(12,13)$. To reveal whether fatostatin suppressed EC through the SREBP metabolic pathway, we first examined the transcriptional expression levels of SREBPs. As we hypothesized, following treatment with fatostatin for $24 \mathrm{~h}$, the expression levels of nuclear SREBP-1 and SREBP-2 were decreased in a dose-dependent manner in Ishikawa and HEC-1A cells, while the precursor SREBP-1 and SREBP-2 levels were mildly changed, showing no statistical significance (Fig. 6A-C). Then, we examined the transcriptional expression of the following SREBP-controlled anabolic genes in Ishikawa and HEC-1A cells: ACL, FASN and SCD-1, which are involved in lipogenesis; HMGCS1, HMGCR, MVK, MVD and LDLR, which are involved in cholesterogenesis; and INSIG1 and SCAP, which are two chaperones. The mRNA expression levels of these genes were significantly downregulated in the fatostatin-treated cells compared with those in the vehicle-treated cells (Fig. 7). Similar results were obtained by western blot analysis. The protein levels of FASN and HMGCR were decreased by fatostatin in a dose-dependent pattern (Fig. 6A-C). Collectively, these results indicated that fatostatin 
A

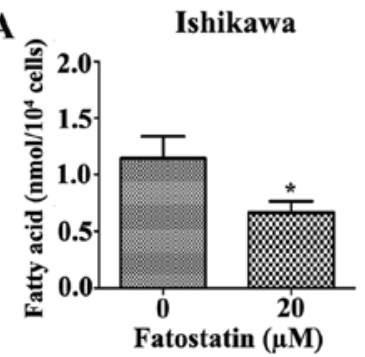

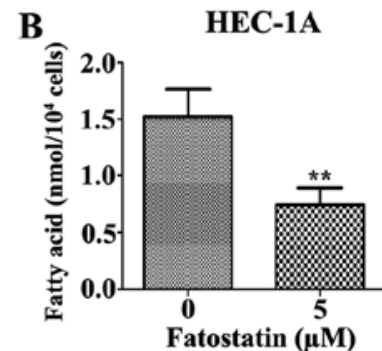
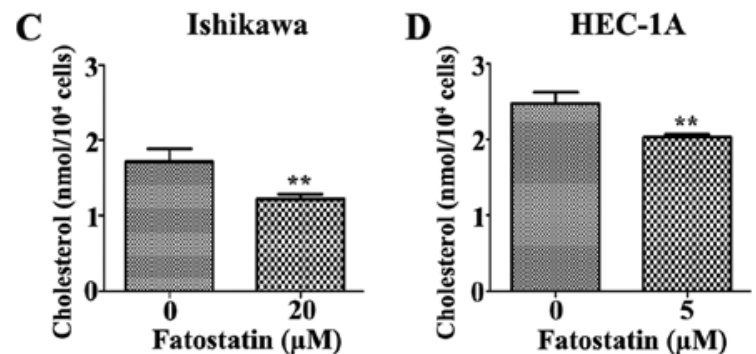

Figure 8. (A and B) Fatostatin significantly decreased the levels of intracellular free fatty acids in Ishikawa and HEC-1A cells compared with those in the vehicle-treated cells. (C and D) Fatostatin significantly decreased the levels of intracellular total cholesterol in Ishikawa and HEC-1A cells compared with the vehicle-treated cells. ${ }^{*} \mathrm{P}<0.05$ and ${ }^{* *} \mathrm{P}<0.01$

significantly inhibited the SREBP metabolic pathways in EC cells.

Since fatostatin suppressed several key genes that were associated with lipogenesis and cholesterogenesis through SREBPs, we performed quantification assays using a free fatty acid quantification detection kit and total cholesterol quantification detection kit to assess the changes in intracellular free fatty acids and total cholesterol, respectively, that were induced by 48 -h treatments with fatostatin. As we hypothesized, the level of intracellular free fatty acids was significantly decreased by fatostatin in Ishikawa and HEC-1A cells compared with that in the vehicle-treated cells (Fig. 8A and B). Similar results were observed for the total cholesterol levels. Fatostatin significantly decreased the level of intracellular total cholesterol in Ishikawa and HEC-1A cells compared with that in the vehicle-treated cells (Fig. 8C and D). Therefore, these results indicated that fatostatin significantly decreased the levels of intracellular free fatty acid and total cholesterol through inhibition of SREBPs in EC cells.

\section{Discussion}

Accumulating evidence has shown that tumor cells reprogram their metabolic pathways to sustain higher proliferative rates, enhance tumor growth and resist cell death signals $(17,18)$. In cancer cells, lipid metabolism is increased to meet high metabolic demands $(18,19)$. Disordered lipid metabolism contributes to different aspects of tumorigenesis. In EC, lipid metabolism is the most upregulated metabolic pathway and impacts the outcome of treatment and/or disease progression in patients with type I EC (20). SREBPs are central regulators of lipid homeostasis and function by transcriptionally activating genes that are involved in fatty acid and cholesterol homeostasis (5). Several studies have reported that SREBPs function as oncogenes in various malignant tumors and that they can promote tumor progression by regulating lipogenesis (6-9). Li et al demonstrated that the expression of SREBP-1 was significantly elevated in EC compared with that in healthy endometrium, that the expression levels were positively correlated with cancer progression, and that knockdown of SREBP-1 expression in EC cells suppressed cell proliferation, reduced clonogenic capacity and induced apoptosis in vitro and in vivo (10). Thus, SREBP-1 functions as an oncogene in EC and can promote EC progression by regulating lipid metabolism, suggesting SREBP-1 as a novel therapeutic target for EC treatment.
SREBPs are membrane-bound, basic helix-loophelix-leucine zipper (HLH-LZ) transcription factors (5). SREBPs are subject to complex post-translational regulation and SCAP is a critical regulator of this process (21). Precursor SREBPs, which associate with two chaperone proteins, namely, INSIG and SCAP, are embedded in the ER. SCAP and INSIG bind to ER membrane-associated cholesterol or oxysterols molecules via sterol-sensing domains that are sensitive to ER membrane sterol levels. When ER membrane sterol levels decrease, INSIG and SCAP undergo conformational changes, and the SCAP/SREBP complex is released from INSIG (22). Then, the SREBP/SCAP complex is escorted from the ER to the Golgi by binding to Sec24, which is a component of the Sar1/Sec23/Sec24 complex of the COPII protein coat $(22,23)$. In the Golgi, SREBPs are released from SCAP and cleaved via site- 1 and site- 2 proteases to generate nuclear SREBPs. Subsequently, nuclear SREBPs activate target genes by binding to sterol response elements and maintain fatty acid and cholesterol homeostasis (21).

Fatostatin is a chemical inhibitor of the SREBP pathway. Fatostatin interacts directly with SCAP at a distinct domain from the sterol-binding site and blocks the ER exit of SCAP and the ER-to-Golgi transport of SREBPs $(12,13)$. Thus, fatostatin can decrease the expression levels of nuclear SREBPs and their downstream genes and subsequently decrease lipid metabolism. As was aforementioned, Li et al investigated the role of SREBP-1 in EC and demonstrated that SREBP-1 was essential for EC cell growth both in vitro and in vivo as determined by immunohistochemistry staining, cell transfection and transduction and subcutaneous tumor implantation. Their results revealed that SREBP-1 functions as an oncogene in EC and may be a novel therapeutic target for EC treatment (10). Therefore, blocking SREBP-regulated metabolic pathways via pharmacological intervention may be a novel therapeutic approach for treating EC. However, they did not perform any further studies on this topic. In the present study, we speculated that fatostatin, which is a chemical inhibitor of the SREBP pathway and can block SREBP-regulated metabolic pathways, may be a novel therapeutic approach for EC treatment, and aimed to investigate the antitumor effects of fatostatin against EC.

Studies have demonstrated that fatostatin promotes a significant reduction in nuclear SREBPs and their downstream genes in prostate cancer and pancreatic cancer cells (14-16). Ankur et al determined that the anticancer properties of fatostatin were not only due to its inhibition of SREBPs and 
effects on lipid metabolism but also attributed to its inhibition of cell division (24). Li et al determined that, in prostate cancer cells, fatostatin inhibited cell proliferation, invasion and migration, promoted G2/M cell cycle arrest and induced caspase-mediated apoptosis. The authors also demonstrated that, in prostate cancer cells, fatostatin suppressed SREBP processing, SREBP transcriptional activity, several key enzymes for lipogenesis and cholesterogenesis, and fatty acid and cholesterol levels $(14,15)$. Siqingaowa et al demonstrated that fatostatin decreased pancreatic cancer cell viability and proliferation (16). In our present study of EC cells, fatostatin significantly decreased the expression of nuclear SREBPs and their downstream genes and reduced free fatty acid and total cholesterol levels. In addition, fatostatin inhibited EC cell viability and colony formation, decreased the invasive and migratory capacities of EC cells, induced G2/M EC cell cycle arrest and promoted caspase-mediated apoptosis in EC cells. Therefore, our study revealed that fatostatin exhibited an antitumor effect by blocking SREBP-regulated lipid metabolic pathways in EC.

Lipid homeostasis is important for maintaining cellular structure and normal function. Fatty acids play essential roles in multiple cellular processes (25), are essential constituents of all biological membrane lipids, and are important substrates for energy storage and metabolism $(25,26)$. FASN, ACL and SCD-1 are three rate-limiting enzymes involved in the biosynthesis of long-chain fatty acids (14). SREBP-1 functions as the key regulator of fatty acid metabolism by transcriptionally regulating the expression of these three lipogenic genes $(5,14)$. Studies have demonstrated that SREBP-1 and FASN play crucial roles in the processes of EC oncogenesis and progression (10,27). ACL expression and activity are markedly increased in cancer cells, including EC cells, and ACL inhibition can suppress tumor cell growth $(20,28)$. Evidence has shown that SCD-1 can enhance tumorigenesis by accelerating the cancer cell proliferation rate, increasing cell invasiveness, and enhancing cell survival (29). Our results revealed that fatostatin markedly suppressed the expression of FASN, ACL and SCD-1 and significantly reduced the levels of intracellular fatty acids in EC cells. The inhibitory mechanism of fatostatin could be attributed to decreasing SREBP-1 transcriptional activity, since SREBP-1 has been demonstrated to transcriptionally regulate the expression of these three lipogenic genes and fatostatin is a chemical inhibitor of the SREBP pathway. These data revealed that fatostatin decreased the expression of cancer-associated lipogenic genes and inhibited EC growth, oncogenesis and progression in vitro by interrupting SREBP-1regulated fatty acid metabolism.

Cholesterol is a major component of lipid rafts, which exhibit a special structure on the cellular membrane and consist of cholesterol and sphingolipids (30). Several kinase receptors and signaling molecules, such as EGFR and AK, are located in lipid rafts $(31,32)$. Therefore, the maintenance of the intracellular cholesterol level is important for maintaining lipid-raft-mediated survival signaling pathways, cell morphology and cell function (30). Studies have suggested that SREBP-2 is a major regulator of cholesterol metabolism and that SREBP-2 upregulates several important cholesterogenic genes, such as HMGCS1, HMGCR and $\operatorname{LDLR}(5,14)$. Although the importance of SREBP-2 in cancer cells and oncogenesis remains relatively unexplored, unlike SREBP-1, a combination knockdown of SREBP-2 and SREBP-1 in cancer cell lines causes EC stress and induces apoptosis in lipoprotein-depleted conditions, and the simultaneous targeting of HMGCR and SREBP-2 is a promising novel antitumor therapeutic strategy (33). HMGCR is the action target of statins and is used to treat high cholesterol levels. Multiple studies have demonstrated that the use of statins is associated with decreased incidences of cancer and decreased deaths from cancers of the breast, colon, pancreas, gastrointestinal tract, liver, endometrium, and ovaries. Statin treatment has the ability to inhibit various cancer processes, including tumorigenesis, growth, angiogenesis, and metastasis (34). In addition, statins have anti-proliferative and anti-metastatic effects on EC cell lines in vitro $(33,35)$. These data demonstrated that HMGCR inhibition has antitumor effects. HMGCS1 is upstream of HMGCR and synthesizes HMG-CoA, the substrate of HMGCR. Analysis of cancer genomic datasets using cBioPortal revealed that HMGCS1 can be amplified in various cancers. HMGCS1 and SREBP-2 knockdown, together with statin-mediated HMGCR inhibition, can robustly enhance tumor cell apoptosis (33). In the present study, we demonstrated that fatostatin markedly decreased the expression levels of these cholesterogenic genes, including HMGCR and HMGCS1, and significantly decreased the total intracellular cholesterol level in EC cells, which is important for maintaining lipid-raft-mediated survival signaling pathways, cell morphology and cell function. The inhibitory mechanism of fatostatin could be attributed to decreasing SREBP-2 transcriptional activity, since SREBP-2 has been demonstrated to transcriptionally regulate the expression of these cholesterogenic genes and fatostatin is a chemical inhibitor of the SREBP pathway. These data revealed that fatostatin decreased the expression of cholesterogenic genes and exhibited an antitumor effect against EC in vitro by interrupting the SREBP-2-regulated cholesterogenic pathway.

Apoptosis is a regulated cellular suicide mechanism, with characteristics including nuclear condensation, cell shrinkage, membrane blebbing, and DNA fragmentation (36). The caspase family consists of the central regulators of apoptosis and contains two types of caspase enzymes, namely, initiator and executioner caspases. Initiator caspases (including caspase-2, caspase-8, caspase-9, caspase-10, caspase-11, and caspase-12) are closely correlated to pro-apoptotic signals. Once activated, full-length caspases are cleaved to form cleaved-caspases and activate downstream effector caspases (including caspase-3, caspase-6, and caspase-7), which in turn induce apoptosis (37). Studies have demonstrated that PARP is an intracellular 'death substrate' and that cleavage of PARP by caspases is likely a prerequisite for the induction of apoptosis in various cells (38). In our present study, we demonstrated that fatostatin decreased the expression levels of full-length caspase-9, caspase- 3 and PARP, increased the expression levels of cleaved caspase-9, cleaved caspase- 3 and cleaved PARP in EC cells, and increased EC cell apoptosis. These data revealed that fatostatin induced caspase-dependent apoptosis in EC cells. Since the potential signaling pathways between fatostatin and the caspase family in EC and the potential underlying molecular mechanisms by which fatostatin decreases the invasive and migratory capacities of EC cells are not fully understood and no in vivo tests were performed, more studies are warranted. 
In summary, in the present study we revealed the potential underlying molecular mechanisms by which fatostatin suppressed EC growth and tumorigenesis. We provided evidence that fatostatin inhibits cell viability and colony formation, decreases the invasive and migratory capacities of EC cells and enhances EC cell apoptosis by blocking SREBP-regulated metabolic pathways. In brief, we demonstrated that fatostatin displayed high antitumor effects against EC in vitro by blocking SREBP-regulated metabolic pathways. Although several underlying mechanisms require further investigation, our findings revealed that fatostatin can be a novel therapeutic strategy for the treatment of EC.

\section{Acknowledgements}

This study was supported by a grant from the National Natural Science Foundation of China (no. 81372808).

\section{Competing interests}

The authors declare that they have no competing interests.

\section{References}

1. Siegel RL, Miller KD and Jemal A: Cancer statistics, 2017. CA Cancer J Clin 67: 7-30, 2017.

2. Suri V and Arora A: Management of endometrial cancer: A review. Rev Recent Clin Trials 10: 309-316, 2015.

3. Gunderson CC, Fader AN, Carson KA and Bristow RE: Oncologic and reproductive outcomes with progestin therapy in women with endometrial hyperplasia and grade 1 adenocarcinoma: A systematic review. Gynecol Oncol 125: 477-482, 2012

4. Hahn HS, Yoon SG, Hong JS, Hong SR, Park SJ, Lim JY, Kwon YS, Lee IH, Lim KT, Lee KH, et al: Conservative treatment with progestin and pregnancy outcomes in endometrial cancer. Int J Gynecol Cancer 19: 1068-1073, 2009.

5. Horton JD, Goldstein JL and Brown MS: SREBPs: Activators of the complete program of cholesterol and fatty acid synthesis in the liver. J Clin Invest 109: 1125-1131, 2002.

6. Li JN, Mahmoud MA, Han WF, Ripple M and Pizer ES: Sterol regulatory element-binding protein-1 participates in the regulation of fatty acid synthase expression in colorectal neoplasia. Exp Cell Res 261: 159-165, 2000.

7. Swinnen JV: Increased lipogenesis in steroid-responsive cancer cells: Mechanisms of regulation, role in cancer cell biology and perspectives on clinical applications. Verh K Acad Geneeskd Belg 63: 321-333, 2001

8. Yang Yu, Morin PJ, Han WF, Chen T, Bornman DM, Gabrielson EW and Pizer ES: Regulation of fatty acid synthase expression in breast cancer by sterol regulatory element binding protein-1c. Exp Cell Res 282: 132-137, 2003.

9. Yahagi N, Shimano H, Hasegawa K, Ohashi K, Matsuzaka T, Najima Y, Sekiya M, Tomita S, Okazaki H, Tamura Y, et al: Co-ordinate activation of lipogenic enzymes in hepatocellular carcinoma. Eur J Cancer 41: 1316-1322, 2005.

10. Li W, Tai Y, Zhou J, Gu W, Bai Z, Zhou T, Zhong Z, McCue PA, Sang N, Ji JY, et al: Repression of endometrial tumor growth by targeting SREBP1 and lipogenesis. Cell Cycle 11: 2348-2358, 2012.

11. Eberhard Y, Gronda M, Hurren R, Datti A, Maclean N, Ketela T, Moffat J, Wraba JL and Schimmer AD: Inhibition of SREBP1 sensitizes cells to death ligands. Oncotarget 2: 186-196, 2011.

12. Kamisuki S, Mao Q, Abu-Elheiga L, Gu Z, Kugimiya A, Kwon Y, Shinohara T, Kawazoe Y, Sato S, Asakura K, et al: A small molecule that blocks fat synthesis by inhibiting the activation of SREBP. Chem Biol 16: 882-892, 2009.

13. Shao W, Machamer CE and Espenshade PJ: Fatostatin blocks ER exit of SCAP but inhibits cell growth in a SCAP-independent manner. J Lipid Res 57: 1564-73, 2016.
14. Li X, Chen YT, Hu P and Huang WC: Fatostatin displays high anti-tumor activity in prostate cancer by blocking SREBP-regulated metabolic pathways and androgen receptor signaling. Mol Cancer Ther 13: 855-866, 2014.

15. Li X, Wu JB, Chung LW and Huang WC: Anti-cancer efficacy of SREBP inhibitor, alone or in combination with docetaxel, in prostate cancer harboring p53 mutations. Oncotarget 6: 41018-41032, 2015.

16. Siqingaowa, Sekar S, Gopalakrishnan V and Taghibiglou C: Sterol regulatory element-binding protein 1 inhibitors decrease pancreatic cancer cell viability and proliferation. Biochem Biophys Res Commun 488: 136-140, 2017.

17. Tennant DA, Durán RV and Gottlieb E: Targeting metabolic transformation for cancer therapy. Nat Rev Cancer 10: 267-277, 2010.

18. Ward PS and Thompson CB: Metabolic reprogramming: A cancer hallmark even warburg did not anticipate. Cancer Cell 21: 297-308, 2012.

19. Deberardinis RJ, Sayed N, Ditsworth D and Thompson CB: Brick by brick: Metabolism and tumor cell growth. Curr Opin Genet Dev 18: 54-61, 2008.

20. Byrne FL, Poon IK, Modesitt SC, Tomsig JL, Chow JD, Healy ME, Baker WD, Atkins KA, Lancaster JM, Marchion DC, et al: Metabolic vulnerabilities in endometrial cancer. Cancer Res 74: 5832-5845, 2014.

21. Williams KJ, Argus JP, Zhu Y, Wilks MQ, Marbois BN, York AG, Kidani Y, Pourzia AL, Akhavan D, Lisiero DN, et al: An essential requirement for the SCAP/SREBP signaling axis to protect cancer cells from lipotoxicity. Cancer Res 73: 2850-2862, 2013.

22. Radhakrishnan A, Goldstein JL, McDonald JG and Brown MS: Switch-like control of SREBP-2 transport triggered by small changes in ER cholesterol: A delicate balance. Cell Metab 8: 512-521, 2008.

23. Goldstein JL, DeBose-Boyd RA and Brown MS: Protein sensors for membrane sterols. Cell 124: 35-46, 2006.

24. Gholkar AA, Cheung K, Williams KJ, Lo YC, Hamideh SA, Nnebe C, Khuu C, Bensinger SJ and Torres JZ: Fatostatin inhibits cancer cell proliferation by affecting mitotic microtubule spindle assembly and cell division. J Biol Chem 291: 17001-17008, 2016.

25. Johannes VS, Koen B and Guido V: Increased lipogenesis in cancer cells: New players, novel targets. Curr Opin Clin Nutr Metab Care 9: 358-365, 2006.

26. Menendez JA and Lupu R: Fatty acid synthase and the lipogenic phenotype in cancer pathogenesis. Nat Rev Cancer 7: 763-777, 2007.

27. Qiu C, Dongol S, Lv QT, Gao X and Jiang J: Sterol regulatory element-binding protein-1/fatty acid synthase involvement in proliferation inhibition and apoptosis promotion induced by progesterone in endometrial cancer. Int J Gynecol Cancer 23: 1629-1634, 2013.

28. Hatzivassiliou G, Zhao F, Bauer DE, Andreadis C, Shaw AN, Dhanak D, Hingorani SR, Tuveson DA and Thompson CB: ATP citrate lyase inhibition can suppress tumor cell growth. Cancer Cell 8: 311-321, 2005.

29. Igal RA: Stearoyl-CoA desaturase-1: A novel key player in the mechanisms of cell proliferation, programmed cell death and transformation to cancer. Carcinogenesis 31: 1509-1515, 2010.

30. Guo D, Bell EH and Chakravarti A: Lipid metabolism emerges as a promising target for malignant glioma therapy. CNS Oncol 2: 289-299, 2013.

31. Freeman MR, Cinar B, Kim J, Mukhopadhyay NK, Di Vizio D, Adam RM and Solomon KR: Transit of hormonal and EGF receptor-dependent signals through cholesterol-rich membranes. Steroids 72: 210-217, 2007.

32. Lingwood D and Simons K: Lipid rafts as a membrane-organizing principle. Science 327: 46-50, 2010.

33. Pandyra AA, Mullen PJ, Goard CA, Ericson E, Sharma P, Kalkat M, Yu R, Pong JT, Brown KR, Hart T, et al: Genome-wide RNAi analysis reveals that simultaneous inhibition of specific mevalonate pathway genes potentiates tumor cell death. Oncotarget 6: 26909-26921, 2015.

34. Wiemer AJ, Hohl RJ and Wiemer DF: The intermediate enzymes of isoprenoid metabolism as anticancer targets. Anticancer Agents Med Chem 9: 526-542, 2009.

35. Nevadunsky NS, Van Arsdale A, Strickler HD, Spoozak LA, Moadel A, Kaur G, Girda E, Goldberg GL and Einstein MH: Association between statin use and endometrial cancer survival. Obstet Gynecol 126: 144-150, 2015. 
36. Indran IR, Tufo G, Pervaiz S and Brenner C: Recent advances in apoptosis, mitochondria and drug resistance in cancer cells. Biochim Biophys Acta 1807: 735-745, 2011.

37. Vyas VK, Chintha C and Pandya MR: Biology and medicinal chemistry approaches towards various apoptosis inducers. Anticancer Agents Med Chem 13: 433-455, 2013.
38. Soldatenkov VA and Potaman VN: DNA-binding properties of poly(ADP-ribose) polymerase: A target for anticancer therapy. Curr Drug Targets 5: 357-365, 2004 